\title{
Diferencias entre áreas científicas en las prácticas de divulgación de la investigación: un estudio empírico en el CSIC
}

\author{
Julia Olmos-Peñuela*; Elena Castro-Martínez*; Manuel Fernández-Esquinas** \\ * INGENIO (CSIC-Universitat Politècnica de València) \\ ** Instituto de Estudios Sociales Avanzados (CSIC) \\ Correo-e: juolpe@ingenio.upv.es; ecastrom@ingenio.upv.es; mfernandez@iesa.csic.es
}

Recibido: 31-05-2013; $2^{a}$ version: 17-12-2013; Aceptado: 17-01-2014.

Cómo citar este artículo/Citation: Olmos-Peñuela, J.; Castro-Martínez, E.; Fernández-Esquinas, M. (2014). Diferencias entre áreas científicas en las prácticas de divulgación de la investigación: un estudio empírico en el CSIC. Revista Española de Documentación Científica, 37(2):e040. doi: http://dx.doi.org/10.3989/redc.2014.2.1096

Resumen: En las últimas décadas se han comenzado a desarrollar políticas gubernamentales e institucionales para la promoción de la divulgación del conocimiento científico. Este artículo explora la implicación de los investigadores en diversos tipos de actividades de divulgación, para identificar si se producen diferencias entre las diferentes áreas científicas. El estudio analiza una muestra amplia de investigadores pertenecientes a las ocho áreas científicas del Consejo Superior de Investigaciones Científicas (CSIC), a los que se ha realizado una encuesta sobre su participación en distintas actividades de divulgación. Mediante un análisis factorial se han construido dos indicadores que agrupan, respectivamente, las actividades de divulgación de carácter individual e institucional. Un contraste ANOVA permite identificar diferencias entre investigadores de ciencias humanas y sociales e investigadores de las ciencias experimentales, pero no muy acusadas. Los resultados pueden ser de interés para el enfoque futuro de las acciones de fomento de la divulgación de la ciencia.

Palabras clave: Divulgación de la ciencia; organismos públicos de investigación; investigadores; culturas de investigación; áreas científicas.

\section{Differences in research dissemination practices between scientific fields: an empirical study of the CSIC}

\begin{abstract}
A range of governmental and institutional scientific policies have emerged in recent decades to promote the dissemination of scientific knowledge throughout society. This article explores the involvement of researchers in various types of dissemination and identifies practices and differences among scientific fields. The study analyses a large sample of researchers from eight scientific fields of the Spanish Council for Scientific Research (CSIC), who were surveyed about their participation in diverse dissemination activities. Two indicators related to individual and institutional dissemination activities were constructed from a factor analysis. The ANOVA test indicates different dissemination patterns between researchers in social sciences and humanities and those from experimental science fields, though the difference is not very marked. These results may be of interest for the future focus of actions to promote scientific dissemination.
\end{abstract}

Keywords: Science dissemination; public research organizations; researchers; research cultures; scientific fields.

Copyright: (c) 2014 CSIC. Este es un artículo de acceso abierto distribuido bajo los términos de la licencia Creative Commons Attribution-Non Commercial (by-nc) Spain 3.0. 


\section{INTRODUCCIÓN}

La velocidad y la magnitud de los cambios científicos y tecnológicos y su impacto en la vida cotidiana justifican que los contenidos de la ciencia y la tecnología deban formar parte de la cultura de los ciudadanos. Sin estas habilidades culturales estarían condenados a no poder interpretar las implicaciones económicas y sociales de la ciencia y la tecnología, a no tener criterio para decidir sobre los interrogantes que plantean los nuevos hallazgos científicos y a no saber valorar el alcance y los efectos de muchos de los nuevos productos y servicios que ofrece el mercado. Todo ello justifica que las políticas científicas -a todos los niveles- contemplen acciones para favorecer la integración de la labor científica y tecnológica en el tejido social, fomentando la reflexión y el debate sobre la relación de la ciencia y la tecnología con la sociedad y la cultura (Comisión Europea, 2006; CICYT, 2008; FECYT, 2011). En este proceso de disminución del déficit de cultura científica de los ciudadanos, los propios investigadores son actores fundamentales (Kunth, 1992; Weigold, 2001). Son ellos los que poseen el conocimiento de mayor nivel sobre los avances científicos en sus respectivos campos. Y más aún, la divulgación de sus conocimientos forma parte de su misión como investigadores financiados con fondos públicos (OCDE, 1996).

Diversos autores han abordado la participación de los investigadores en actividades de divulgación, sus motivaciones y sus determinantes (Jensen y otros, 2008; Jensen, 2011; Torres-Albero y otros, 2011, entre otros), y han indagado sobre las barreras y los factores que favorecen el nivel de implicación (Poliakoff y Webb, 2007; MartínSempere y otros, 2008). Una hipótesis relevante se refiere a las diferencias y condicionantes procedentes de los campos científicos (Jensen, 2011; Kingston, 2012). Diversos autores atribuyen distinta frecuencia y distintas prácticas de divulgación a las disciplinas experimentales y a las disciplinas humanísticas y sociales, y describen las razones que lo justifican. Se ha discutido la existencia de distintas motivaciones para involucrarse en divulgación procedentes de las formas de entender la tarea de investigación (Besley y otros, 2012). Otros autores atribuyen las diferencias a la distinta naturaleza del trabajo de investigación, así como a la existencia de distintas demandas por parte del entorno (Bauer y Jensen, 2011). Sin embargo, pocos estudios disponen de una medida estandarizada de la amplia gama de actividades de divulgación en una diversidad de disciplinas científicas que permita observar detalladamente las diferencias entre áreas. Tampoco se han realizado exploraciones basadas en metodologías cuantitativas dirigidas a construir indicadores sobre los que poder probar empíricamente dichas diferencias.

Este artículo analiza las actividades de divulgación de la ciencia realizadas por un conjunto amplio de investigadores pertenecientes a diversas áreas de investigación. Los objetivos principales son indagar la frecuencia con que los investigadores participan en una variedad de canales de divulgación e identificar hasta qué punto existen diferencias entre áreas en dicho conjunto de actividades. Este estudio utiliza una fuente de datos y un procedimiento de análisis que permite observar de manera detallada los comportamientos relacionados con la divulgación, en concreto, una encuesta realizada a 1.583 investigadores del Consejo Superior de Investigaciones Científicas (CSIC) pertenecientes a las ocho áreas de conocimiento en las que se estructura este organismo.

\section{LAS MÚLTIPLES DINÁMICAS DE LAS ACTIVIDADES DE DIVULGACIÓN}

En las llamadas "sociedades del conocimiento" los investigadores desempeñan una diversidad de roles relacionados con las capacidades asociadas al trabajo científico. En primer lugar, actúan frecuentemente como expertos (Kalleberg, 2000) en asuntos de relevancia social en medios de comunicación o en eventos con repercusión pública. La emisión de juicios basados en la experiencia en un campo científico puede contribuir a configurar la opinión pública o a orientar el comportamiento de la ciudadanía. Además, como principales conocedores de sus especialidades científicas y tecnológicas en que se organiza la producción de conocimiento, los investigadores desempeñan un papel relevante en la formación científica, tanto directamente como mediante su colaboración en la actualización del conocimiento del profesorado, en la educación científica, así como en la elaboración de materiales didácticos, tales como libros de texto o materiales audiovisuales, etc. En tercer lugar, los investigadores actúan como transmisores de los descubrimientos científicos y desempeñan un papel cívico (Kyvic, 2005). Algunos de esos hallazgos merecen conocerse debido a su trascendencia para interpretar la naturaleza de los fenómenos o a sus implicaciones prácticas para resolver problemas sociales y económicos. Por ello, los investigadores suelen ser interlocutores privilegiados en los eventos dirigidos a comunicar los descubrimientos, tales como conferencias, ferias o semanas de la ciencia.

Este conjunto de roles se refleja en las múltiples actividades de divulgación (o sus términos sinónimos como diseminación, popularización o comunicación social de la ciencia) dirigidas a llevar las capacidades y hallazgos de la indagación científica a grupos sociales más amplios que los usuarios potenciales directos de los resultados de la investigación (Burns y otros, 2003). Ahora bien, los condicionantes que surgen en la multitud de especialidades del saber pueden dar lugar a un comportamiento diferenciado entre disciplinas. Es habitual pensar que una de las principales diferencias se presenta entre las ciencias experimentales y tecno-científicas y las ciencias humanas y sociales (Olmos-Peñuela y otros, 2013). 
La expresión de "las dos culturas", acuñada por C.P. Snow en 1959 en una conferencia pronunciada en la Universidad de Cambridge (Snow, 1959), se ha convertido en un término popular para referirse a los dos estilos de trabajo intelectual representados por las humanidades y las ciencias experimentales. El trabajo de Snow fue criticado ampliamente, al considerarse una simplificación de la enorme diversidad de la tarea intelectual. En ocasiones, lo que ha trascendido de esta conferencia es la visión vulgar que resalta los prejuicios de los expertos de la tecnociencia respecto a los intelectuales humanistas y viceversa (León, 2011).

A pesar de la controversia, acudir a esta gran división aún resulta de utilidad para enmarcar la discusión referida a las diferencias entre grandes grupos de disciplinas. Para este trabajo conviene quedarse con la intención crítica original de Snow, que apuntaba a las deficiencias sustanciales en la formación de los intelectuales de su época con el ánimo de contribuir a superarlas (Snow, 1963). Se refería principalmente a la diferencia cognitiva y de identidad que contribuía a la falta de diálogo entre disciplinas. Esta diferencia se interpretaba como resultado de la socialización en un sistema de valores específico propio de organizaciones académicas que separaban rígidamente las ciencias experimentales y las humanidades.

La división apuntada por Snow se ha trasladado también a las interpretaciones sobre el trabajo de los científicos, y en particular a las actividades de divulgación. Por una parte, la actividad específica de divulgar se suele vincular al proceso de descubrimiento propio de las disciplinas de las ciencias experimentales (López Cerezo y Verdadero, 2003). A estos científicos frecuentemente se les considera en una "torre de marfil", de la que salen ocasionalmente para comunicar sus hallazgos al público, participando en algunas actividades extra-científicas (Guston y Keniston, 1994). Por otra parte, se atribuye a los investigadores humanísticos una cultura de divulgación enraizada en su forma de entender la función social de sus estudios. Frecuentemente está ligada a la figura del intelectual orgánico surgida sobre todo en los modelos universitarios europeos (Abbott, 2001), a la que se atribuye una vocación por generar interpretaciones del mundo e influir en los asuntos de marcan la agenda política o ciudadana (Beyer y Trice, 1982). Lo cierto es que, mientras que la contribución de las ciencias humanas y sociales a la cultura forma parte del quehacer de los investigadores de las áreas de humanidades y ciencias sociales (Garland, 2012), en el caso de los científicos experimentales es más reciente la constatación de que la ciencia y la tecnología afectan a la vida cotidiana de los ciudadanos y que éstos no deberían ignorar los avances en estos ámbitos ni los efectos de estos avances sobre sus vidas, idea que está en la base del reciente apoyo a la divulgación de la ciencia desde las políticas científicas. Aunque los estudios de Ciencia, Tecnología y Sociedad comenzaron en los Estados
Unidos y el Reino Unido a finales de 1960 y principios de 1970 (López Cerezo y Verdadero, 2003), hasta finales de los años 90 del pasado siglo los responsables de las políticas científicas no comenzaron a implementar programas para promover la cultura científica en la sociedad. En este trabajo es útil centrarse igualmente en el planteamiento crítico dirigido a superar una visión simple de las disciplinas en materia de divulgación. En este sentido, el interés de la tesis de las dos culturas es que proporciona un marco de partida para indagar los condicionantes que llevan a un comportamiento distinto en los grandes grupos disciplinarios, aunque es conveniente considerar la diversidad que puede surgir del actual complejo de especialidades científicas. Las preguntas a las que se pretende dar respuesta en este trabajo son: ¿las grandes divisiones disciplinarias afectan a las prácticas de divulgación de la investigación? ¿Cuáles son estas diferencias? La hipótesis general de partida es que, aunque las disciplinas por sí mismas no determinan la orientación de los investigadores que las practican, las divisiones disciplinarias reúnen pautas de trabajo, comportamientos, relaciones sociales y formas de concebir la tarea científica que pueden afectar a la divulgación.

Para contestar a estas preguntas se parte de tres líneas argumentales que ayudarían a explicar las diferencias y que funcionan como hipótesis provisionales. La primera tiene que ver con la distinta naturaleza del trabajo de investigación en las ciencias experimentales y en las ciencias humanas y sociales, principalmente en dos dimensiones: los rasgos cognitivos del trabajo de investigación y la manera en la que se producen y se entienden los descubrimientos científicos, y los rasgos organizativos referidos a la forma de llevar a cabo la tarea científica. La segunda línea del argumento tiene que ver con la distinta forma de concebir la utilidad de los resultados, así como con la forma de entender la difusión del conocimiento en ambos grupos disciplinarios. La tercera línea del argumento tiene que ver con las distintas influencias y apoyos provenientes del entorno, tanto en lo referido a la demanda de conocimiento como a las exigencias de legitimación de las disciplinas. En particular, una influencia importante puede provenir de la participación de las organizaciones científicas mediante el establecimiento de operativos especializados o mediante la celebración de eventos y también de los responsables de las políticas científicas, desde el momento en que la divulgación ha entrado a formar parte de sus objetivos $y$, por ello, destina recursos al impulso de este tipo de actividades. En algunas actividades de divulgación, las organizaciones son actores que apoyan, canalizan o suplen las carencias de los investigadores, mientras que, en otras actividades, los factores determinantes son sus habilidades y la iniciativa individual. La exposición de estas influencias se realiza en cada una de las grandes divisiones disciplinarias, que se exponen como modelos típicos. 
a) El modelo de divulgación de las ciencias experimentales

En lo referido a la naturaleza del trabajo, una parte importante de las actividades de divulgación está vinculada al descubrimiento, entendido como la culminación del trabajo organizado en torno a proyectos de investigación. La divulgación se asocia frecuentemente a la comunicación de los que se consideran hallazgos relevantes (Delanty, 2001). Estas actividades tienen sentido en las etapas de finalización de los proyectos y suelen formar parte de la secuencia temporal de un trabajo de investigación. Se trata de actividades puntuales que tienen sentido cuando se tiene la certeza de que un descubrimiento se ha producido y ha sido reconocido por las comunidades científicas de referencia, normalmente a través de canales de comunicación especializados, como conferencias y revistas científicas. Por otra parte, los científicos experimentales y los tecnólogos suelen trabajar de manera colectiva. Requieren con más frecuencia de un instrumental especializado y de laboratorios, así como de equipos de personas más numerosos, con perfiles especializados para los que existe una división del trabajo más pronunciada. Las actividades de divulgación son, por tanto, una fase del trabajo correspondiente a un proceso colectivizado (Ziman, 1995).

En lo referido a la utilización de los resultados por parte de los agentes sociales, los hallazgos y capacidades de las ciencias experimentales suelen ser usados por parte de colectivos muy especializados de sectores industriales, particularmente los dependientes de la ciencia (Pavitt, 1984) o por sectores de servicios intensivos en conocimiento. La divulgación en estos casos no forma parte del modelo interiorizado por los investigadores de las ciencias experimentales, que está más vinculado a los usuarios directos de sus capacidades y conocimientos en el ámbito de la economía o la administración pública. Además, en ocasiones el conocimiento pierde valor si es divulgado, por lo que es muy frecuente que los contratos con las empresas excluyan expresamente esta posibilidad sin su autorización expresa (Polt y otros, 2001).

Por último, las demandas del entorno relativas a la divulgación también pueden moldear las actividades de este colectivo. Por un lado, las demandas directas (sobre todo por parte de los medios de comunicación) están relacionadas con problemas y coyunturas muy concretas, en las que se requiere la pericia de un especialista, o bien con hallazgos que tienen especial transcendencia en un problema social o económico de primer orden. Son, por tanto, demandas puntuales asociadas al ámbito de experiencia del investigador. Por otra parte, el modo colectivo de su trabajo está más ligado a las actividades que realizan en el marco de las iniciativas emprendidas por las instituciones con las que trabajan. Estas organizaciones son importantes a la hora de proveer infraestructura y apoyo para tareas a las que los investigadores no se dedican habitualmente y para las que no suelen tener formación especializada (Bauer y Jensen, 2011).

De este conjunto de rasgos se derivan algunas implicaciones importantes para las actividades de divulgación. Es de esperar que los científicos experimentales realicen divulgación principalmente en ciertas fases del trabajo de investigación y que, por tanto, en general participen con menos frecuencia. También es de esperar que realicen más actividades promovidas por el organismo en que trabajan o en colaboración con otros organismos interesados en la promoción de la ciencia. Sus actividades posiblemente estarán más vinculadas a la organización de eventos puntuales que a tareas surgidas de la iniciativa individual.

b) El modelo de divulgación de las ciencias humanas y sociales

En el aspecto cognitivo del trabajo de los investigadores de las ciencias humanas y sociales no existen fases tan claramente asociadas al descubrimiento como en otras disciplinas, dada la distinta forma en que se desarrollan los paradigmas científicos. Ello se debe a que, para los humanistas y los científicos sociales, una parte esencial del material de trabajo es de carácter simbólico y conceptual, y en menor medida instrumental (Amara y otros, 2004). El objeto de numerosas especialidades es elaborar significados o interpretaciones de las realizaciones humanas, tales como los hechos del pasado, las manifestaciones artísticas o los comportamientos sociales. El descubrimiento también se da en estos campos, pero esa no es la etapa final, sino en muchos casos el comienzo de la verdadera labor de investigación e interpretación de los citados hallazgos. En consonancia, la tarea de divulgación no está tan asociada a una fase concreta del proceso cognitivo, sino más bien a la acumulación de conocimiento considerado relevante. En el aspecto de la organización del trabajo también hay rasgos que pueden privilegiar distintas formas de divulgación. Salvo en casos muy específicos, como puede ser la arqueología, en la investigación en ciencias humanas y sociales se requiere en menor medida de instrumental y equipos grandes, y se trabaja en mayor medida con equipos reducidos de personas, cuando no de forma individual.

En lo que concierne a los usos del trabajo de investigación, no están basados en la misma medida en usuarios especializados. Gran parte de los resultados están dirigidos a orientar la acción de públicos más amplios y están motivadas por un objetivo cívico (Kyvic, 2005). En unos casos mediante la construcción de narrativas que proveen de significado a la apreciación de los hechos. En otros casos, mediante la provisión de datos, diagnósticos o interpretaciones de la realidad social que pueden informar la toma de decisiones. Esta forma de utilizar los resultados contribuye a que la divulgación se considere como uno de los componentes fundamentales de la difusión del conocimiento, pues su utilidad va asociada a la necesidad de que el público asimile las interpretaciones. 
Por lo que se refiere a las relaciones con el entorno, los investigadores de ciencias humanas y sociales reciben demandas específicas por parte de los medios de comunicación, relacionadas con el doble rol de "intelectual orgánico" y experto técnico (Kalleberg, 2000; Delanty, 2001). Es frecuente contar con expertos en aquellos problemas habituales en el ámbito político, tales como el alcance del estado del bienestar, la educación, el estado de la economía o los servicios públicos. En particular, en algunas coyunturas de crisis económica o de controversias sociales y políticas, se demanda la participación de expertos con competencias en temas de interés social (inmigración, economía, envejecimiento, etc.). Por otro lado, una faceta importante de la experiencia de los humanistas está asociada al sector cultural, al que proveen de contenidos. Estos contenidos constituyen una parte relevante de los productos culturales que los medios de comunicación orientan al consumo del público educado interesado, por ejemplo, en la historia, el arte o el conocimiento de distintas culturas.

Otra parte de las demandas del entorno puede provenir de la legitimación que se espera de las ciencias humanas y sociales por parte de los organismos de I+D y de las posibles fuentes de financiación. Un criterio de relevancia para justificar las inversiones públicas en salarios e infraestructuras científicas es que el trabajo de los investigadores proporcione conocimientos de utilidad social, mientras que la justificación de las ciencias experimentales suele estar más vinculada a la relevancia de sus actividades para el desarrollo tecnológico o la innovación en los sectores productivos. Adicionalmente, otro elemento importante en las ciencias humanas y sociales es la disposición de ciertas habilidades afines a sus procesos de investigación. Es posible que estos investigadores dispongan de destrezas útiles para la formación y la participación en medios de comunicación, como el manejo de elementos simbólicos tales como la comunicación - la escritura. Esto les permite llevar a cabo de manera autónoma sus actividades de divulgación en contextos en los que el apoyo institucional es menos decisivo.

Como resultado de la confluencia de estos rasgos, es de esperar que los investigadores de ciencias humanas y sociales desarrollen en general más actividades de divulgación y, además, que estas actividades se concentren en mayor medida en canales de participación individual que no necesitan del apoyo institucional, sobre todo en medios de comunicación y en escritos y materiales divulgativos.

\section{EL CONTEXTO}

\subsection{Las políticas para el fomento de la} divulgación científica

A partir de la adopción, por parte de la Conferencia Mundial sobre la Ciencia (UNESCO), de la
"Declaración sobre la ciencia y el uso del conocimiento científico" de 1 de julio de $1999^{1}$, muchos países establecieron iniciativas para promover la difusión social de la ciencia (exposiciones, semanas de la ciencia, ferias científicas, etc.). La primera vez que la UE incluyó este punto fue en el VI Programa Marco de I+D (2002-2006); el Ilamado "Programa de Ciencia y Sociedad", que formaba parte del tema "Estructurando el Espacio Europeo de Investigación", contando con un presupuesto de 88 millones en euros. En el VII Programa Marco de I+D (2007-2013) el programa pasa a denominarse "Ciencia en la Sociedad", incrementado su presupuesto hasta los 330 millones de euros (casi cuatro veces el del período anterior).

Con respecto a España, la primera vez que la política científica nacional contiene previsiones para fomentar la divulgación social de la ciencia es en el Plan Nacional de Investigación Científica, Desarrollo e Innovación Tecnológica 2000-2003, que en 2000 destinó a estos fines 189 millones de pesetas para financiar 37 acciones. En 2001 se crea la Fundación Española para la Ciencia y la Tecnología, entre otros objetivos, para "propiciar el desarrollo de una cultura científica e innovadora de toda la sociedad y actuar como vehículo de comunicación y difusión científica entre sus agentes y de ellos con la sociedad en general" (FECYT, 2003). De acuerdo con los datos de las memorias anuales, la FECYT ha destinado, en el periodo 2008-2012, cerca de 21.000 millones de euros a la financiación de las acciones encuadradas en el programa de ciencia y sociedad (FECYT, varias memorias). Así mismo, la Ley 14/2011, de 1 de junio, de la Ciencia, la Tecnología y la Innovación, actualmente en vigor, en su Artículo 38 "Cultura científica y tecnológica", establece el fomento de la cultura científica y tecnológica a la sociedad, y considera las actividades de divulgación y de cultura científica y tecnológica como consustanciales a la carrera investigadora.

\subsection{El Consejo Superior de Investigaciones Científicas (CSIC) como lugar estratégico de investigación}

EI CSIC es el organismo público de investigación de mayor envergadura del Estado Español. Engloba prácticamente todas las disciplinas científicas, organizadas en ocho áreas del conocimiento (Tabla I). La unidad organizativa principal es el instituto de investigación, en el que trabajan los investigadores en torno a equipos o proyectos de investigación. En el año 2011, el CSIC contaba con 126 institutos distribuidos por todo el territorio español, dirigidos desde la organización central ubicada en Madrid. Ese mismo año, el CSIC contaba con 14.050 empleados, de los cuales 5.375 eran personal científico y 3.122 eran investigadores de plantilla (CSIC, 2012).

Para interpretar la implicación de los investigadores del CSIC en actividades de divulgación es conveniente analizar en qué medida el CSIC tiene en cuenta esta actividad en su política y en sus 
prácticas organizativas. En particular, se consideran cinco dimensiones de las organizaciones que pueden ser importantes para favorecer (o impedir) el compromiso de los investigadores con la divulgación (Jacobson y otros, 2004): a) presencia en las prioridades políticas; b) consideración en los sistemas de selección y de promoción; c) dedicación de recursos; d) dotación de estructuras; y e) documentación de este tipo de actividades, que permita su estandarización y proporcione indicadores para evaluar su desempeño.

Las actividades de divulgación forman parte de la misión múltiple del CSIC, y como tal, están recogidas en su reglamento de $2007^{2}$. Estas actividades son una novedad desde el punto de vista organizativo, dado que en el reglamento anterior, del año 2000, aún no figuraba esta función. En el plan estratégico del organismo para 2006-2009 se incluyó una línea específica para fomentar estas actividades (DIVULGA). No obstante, hasta el plan estratégico 2010-2013 no se han incorporado indicadores de divulgación que permitan valorar el avance en esta materia.

En la actualidad los investigadores tienen libertad para realizar estas tareas, aunque no se incluyen como parte obligatoria de la carga de trabajo. En la batería de indicadores para evaluar el desempeño de los institutos figuran dos que informan sobre las actividades de divulgación (participación en eventos y elaboración de materiales de divulgación). Constituyen uno de los siete aspectos que se valora en la concesión del llamado componente por Méritos Investigadores del Complemento Específico (Quinquenios), ${ }^{3}$ aunque forman parte de un grupo de méritos diverso (servicios, comités editoriales de revistas, etc.). No obstante, la principal barrera es que estas actividades no se valoran en los sistemas de reclutamiento de los científicos (concurso-oposición) ni en los de promoción. Los incentivos para la promoción profesional se basan principalmente en publicaciones indexadas $y$, en algunas especialidades, en la licencia de patentes y desarrollos tecnológicos relevantes. La situación habitual es que los investigadores combinan la divulgación en distinta medida con la captación y ejecución de proyectos, la realización de publicaciones, la transferencia de conocimiento mediante contratos o convenios con empresas o administraciones públicas y las actividades de formación universitaria a tiempo parcial. Ello ocurre en función de la orientación y las oportunidades del investigador, normalmente de acuerdo con los criterios de planificación estratégica existentes en cada centro, aunque los investigadores suelen tener capacidad para decidir en qué tipos de actividades de divulgación quieren involucrarse, así como los esfuerzos que dedican a ellas.

EI CSIC dedica recursos a la divulgación básicamente mediante dos vías. A través de su editorial, que, además de publicaciones científicas, edita publicaciones y producciones audiovisuales dirigidas al gran público y colecciones específicamente dedicadas a la divulgación de la ciencia y la tecnología (Divulgación, Qué sabemos de e Informes). Adicionalmente, consigue recursos de entidades privadas y de convocatorias públicas nacionales y europeas destinadas al fomento de la divulgación para organizar diversos tipos de actividades (semana de la ciencia, ferias, ciclos de conferencias, Ciudad Ciencia, el CSIC en la Escuela, proyectos en torno a conmemoraciones científicas, etc.) en las que participan los investigadores, que también consiguen recursos para llevar a cabo iniciativas propias de sus institutos.

Para organizar las actividades de divulgación propias y coordinar las emprendidas por los institutos, el CSIC se dotó en 2004 de una estructura denominada "Área de cultura científica", que posteriormente se convirtió en la Vicepresidencia Adjunta de Cultura Científica. Esta unidad dispone de personal especializado en la divulgación científica y coordina la labor del personal que se ha ido incorporando a los diversos centros y unidades territoriales gracias a las ayudas públicas, y que constituyen la Red de Cultura Científica del CSIC. No obstante, a excepción de los centros más grandes o con una dimensión social más acusada (como el Museo de Ciencias Naturales o el Real Jardín Botánico), los institutos no suelen disponer de personal ni infraestructuras para la divulgación.

Finalmente, respecto a la documentación, aspecto relevante para poder establecer los procesos y rutinas que permitan medir los avances en la materia, hasta el momento se han establecido dos indicadores de divulgación (eventos y materiales). Se utilizan para evaluar el grado de cumplimiento del plan estratégico de los institutos, aunque es necesario profundizar para lograr indicadores más precisos y robustos. Es conveniente señalar que, en la fecha de la encuesta, el CSIC no registraba las actividades de divulgación realizadas por los investigadores a nivel individual. Por tanto, la única posibilidad de contabilizar y observar este comportamiento de manera sistemática, al margen de los contratos y convenios formales con instituciones externas al CSIC, era la realización de una encuesta. Actualmente la base de datos de contribuciones científicas del organismo (ConCiencia) recopila estos materiales (Bernal y Ponsati Obiols, 2011).

\section{METODOLOGÍA}

\subsection{Fuente de datos, trabajo de campo y muestra}

La población objeto del estudio está formada por todos los investigadores de plantilla del CSIC (en las categorías de científico titular, investigador científico y profesor de investigación), así como por los doctores contratados con cargo a programas públicos de formación o perfeccionamiento (principalmente doctores de los programas JAE-Doc, Juan de la Cierva, Ramón y Cajal y programas similares 
de administraciones públicas). En el momento de extraer los datos (enero del año 2011), en estas categorías trabajaban 4.240 investigadores ${ }^{4}$.

A partir de esta población se ha realizado una encuesta de tipo "multimétodo" que combina la realización de un cuestionario online a través de un sitio web con el seguimiento y refuerzo telefónico llevado a cabo por un grupo de encuestadores profesionales.

Para contactar con los investigadores a los que iba dirigida la encuesta se partió de la dirección de correo electrónico. En la primera fase del trabajo de campo se envió el cuestionario por correo electrónico acompañado de información sobre el estudio e instrucciones detalladas. Durante dos semanas se realizaron recordatorios mediante tres envíos de correo electrónico que dieron lugar a una tasa de respuesta del $23 \%$. En la segunda fase se realizó un seguimiento telefónico a aquellos investigadores que no habían respondido la encuesta. El seguimiento se utilizó como recordatorio para el acceso al cuestionario on-line $y$, al mismo tiempo, se empleó para ofrecer posibilidades alternativas de respuesta por teléfono y por correo postal. Mediante el seguimiento telefónico se realizó una post estratificación dirigida a obtener un reparto proporcional de la muestra de acuerdo con las ocho áreas científicas y las categorías profesionales del CSIC. Para ello, se concentraron las llamadas en aquellos grupos con menor tasa de respuesta en la primera fase hasta obtener desvíos de cada estrato inferiores al 3\%.

El resultado de este procedimiento ha dado lugar a un total de 1.583 encuestas (tasa de respuesta del $37,3 \%$ ), lo que supone un error muestral de $\pm 1,9 \%$ para un nivel de confianza del $95 \%$ en el supuesto de mayor dispersión de $p=0,5$. En la tabla I se muestra la distribución de la población de investigadores del CSIC y la distribución de los investigadores encuestados según las áreas científicas. Las pruebas ji-cuadrado $\left(x^{2}\right)$ realizadas confirman que no hay diferencias significativas entre la distribución de la población y de la muestra por área científica, excepto para el caso de las ciencias agrarias, cuya muestra está sobrerrepresentada.

\subsection{Indicadores sobre actividades de divulgación}

Las actividades de divulgación se han recogido mediante una pregunta que responde al texto "Indique la frecuencia aproximada con la que ha realizado actividades relacionadas con la divulgación o la comunicación social de la ciencia en los últimos tres años". Esta pregunta ofrece nueve posibilidades, incluyendo publicaciones divulgativas, participación en medios de comunicación (prensa, programas de radio o televisión), participación en do-

Tabla I. Población y muestra de los investigadores del CSIC: distribución por área científica

\begin{tabular}{|c|c|c|c|c|c|c|}
\hline \multirow{2}{*}{ Áreas científicas } & Población & Población & Muestra & Muestra & $\%$ Diferencias & $\begin{array}{l}\text { Tasa de } \\
\text { respuesta }\end{array}$ \\
\hline & (N) & $(\%)$ & (N) & $(\%)$ & Prueba $x^{2}$ & $(\%)$ \\
\hline Biología y biomedicina & 771 & $18,2 \%$ & 244 & $15,4 \%$ & $-2,8 \%$ & $31,6 \%$ \\
\hline Ciencia y tecnología de alimentos & 285 & $6,7 \%$ & 128 & $8,1 \%$ & $1,4 \%$ & $44,9 \%$ \\
\hline Ciencia y tecnología de materiales & 562 & $13,3 \%$ & 201 & $12,7 \%$ & $-0,6 \%$ & $35,8 \%$ \\
\hline Ciencia y tecnologías físicas & 569 & $13,4 \%$ & 204 & $12,9 \%$ & $-0,5 \%$ & $35,9 \%$ \\
\hline Ciencia y tecnologías químicas & 480 & $11,3 \%$ & 209 & $13,2 \%$ & $1,9 \%$ & $43,5 \%$ \\
\hline Ciencias agrarias & 412 & $9,7 \%$ & 203 & $12,8 \%$ & $3,1 \%+$ & $49,3 \%$ \\
\hline Recursos naturales & 759 & $17,9 \%$ & 277 & $17,5 \%$ & $-0,4 \%$ & $36,5 \%$ \\
\hline Humanidades y ciencias sociales & 402 & $9,5 \%$ & 117 & $7,4 \%$ & $-2,1 \%$ & $29,1 \%$ \\
\hline TOTAL & 4.240 & & 1.583 & & & $37,3 \%$ \\
\hline
\end{tabular}

La prueba $x^{2}$ permite verificar si hay diferencias entre la población y la distribución de la muestra para cada área de conocimiento. El símbolo ' + ' indica la existencia de diferencias estadísticamente significativas al 5\%, concretamente, la prueba indica que el área de ciencias agrícolas está sobrerepresentada en la muestra. 
cumentales científicos, elaboración de contenidos web divulgativos, conferencias o mesas redondas de divulgación, cursos y conferencias en colegios o centros de enseñanza, participación en semanas o ferias de la ciencia, y participación en eventos de puertas abiertas del CSIC o eventos similares. La frecuencia de estas actividades se ha medido a través de una escala ordinal de cuatro posibles respuestas, que comprenden las categorías de "nunca" (ninguna vez), "en algunas ocasiones" (1-3 veces), "a menudo" (4-6 veces) y "muy a menudo" (7 veces o más).

En los estudios realizados hasta la fecha no existe consenso sobre el periodo de referencia a utilizar para contabilizar las actividades de divulgación de los investigadores, lo que genera problemas de comparabilidad entre países y colectivos. Los periodos suelen variar entre 5 y 2 años (Weigold, 2001). En este caso, la elección de tres años se debe a las posibilidades de recuerdo para enmarcar una encuesta. Los pretest realizados indicaban que el recuerdo se reducía en periodos superiores a los tres años.

\section{RESULTADOS}

\subsection{Análisis descriptivos}

En la figura 1 se puede observar la diversidad de actividades en la que los investigadores han participado. Los resultados indican que menos del $6 \%$ de los investigadores no ha participado en ninguna actividad de divulgación en los últimos tres años. Este dato confirma resultados previos de estudios realizados en el contexto español: un $4,4 \%$ del personal científico-investigador del CSIC afirma no haber realizado ninguna actividad de di- vulgación (Martín-Sempere y otros, 2004; TorresAlbero y otros, 2011), elevándose la cifra hasta un $16 \%$ para el caso de profesores universitarios (Rey-Rocha y otros, 2006; Torres-Albero y otros, 2011). Estos datos contrastan con el caso francés donde los investigadores del CNRS presentaban tasas de inactividad superiores al $40 \%$. Así mismo, la figura 1 muestra que más de la mitad de la muestra $(61 \%)$ ha participado en 4 o más actividades diferentes en el periodo de referencia, siendo la pauta más común la realización de entre 3 y 5 actividades distintas.

Por su parte, en la tabla II aparece la frecuencia (intensidad) para cada una de las nueve actividades de divulgación. En términos generales, la participación de investigadores en actividades de divulgación no es muy frecuente en la mayoría de los casos: los porcentajes de investigadores que afirma no haber participado en una actividad de divulgación específica se encuentran en el intervalo entre $22,9 \%$ (publicaciones en libros de texto y revistas de divulgación) y $68,7 \%$ (participación en documentales u otros videos científicos). Los resultados presentados en la figura 1 y tabla II son consistentes y coherentes entre ellos dado que, mientras que en el primer caso (figura 1 ) se realiza un análisis muy "restrictivo", al considerar el porcentaje de investigadores que no participan en ninguna actividad (es decir que responden que "nunca" han participado en ninguna de las 9 actividades consideradas), en el segundo de los casos (tabla II) se analiza la intensidad de participación actividad por actividad, de forma individual, razón por la cual es razonable obtener porcentajes significativamente superiores.

Figura 1. Distribución del número de actividades de divulgación distintas en las que un investigador ha participado al menos una vez en los últimos tres años

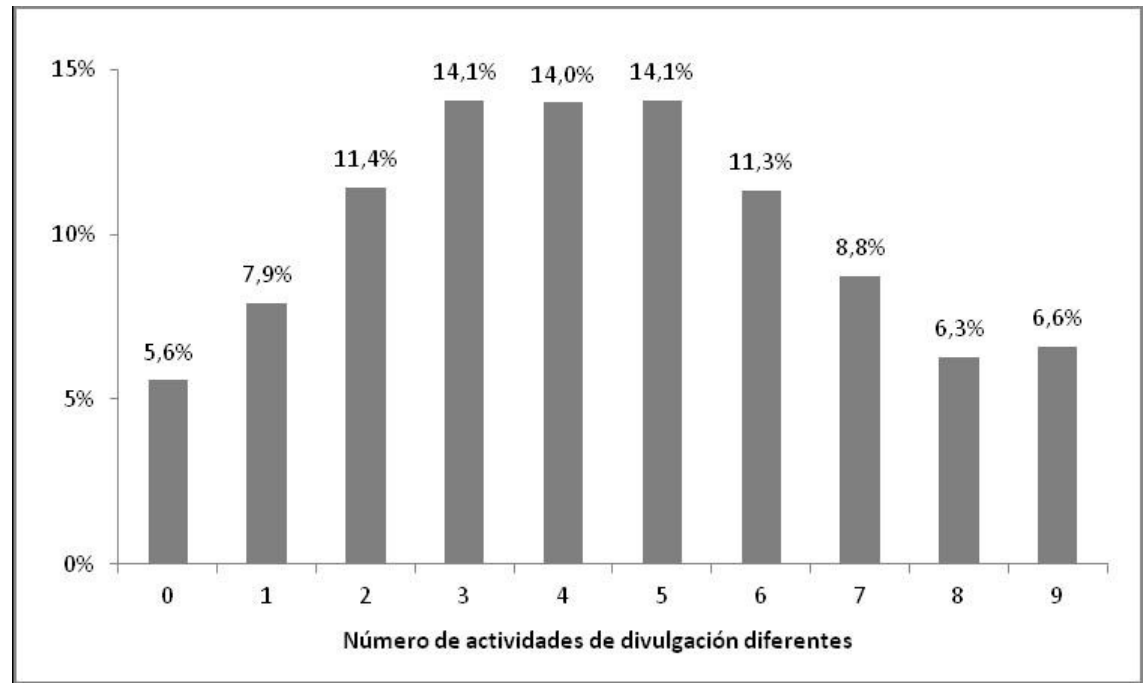


Tabla II. Distribución (\%) de la participación de los investigadores del CSIC en actividades de divulgación en los últimos 3 años

\begin{tabular}{|c|c|c|c|c|}
\hline \multirow{2}{*}{ Actividades de divulgación } & \multirow{2}{*}{ Nunca } & \multirow{2}{*}{$\begin{array}{c}\text { En algunas } \\
\text { ocasiones }\end{array}$} & \multirow{2}{*}{$\begin{array}{c}\text { A menudo } \\
\text { (4-6 veces) }\end{array}$} & \multirow{2}{*}{$\begin{array}{l}\text { Muy a menudo } \\
\text { (7 o más veces) }\end{array}$} \\
\hline & & & & \\
\hline Publicación de textos en libros y revistas de divulgación & 22,9 & 48,2 & 16,1 & 12,8 \\
\hline $\begin{array}{l}\text { Participación en conferencias o mesas redondas de } \\
\text { divulgación }\end{array}$ & 35,1 & 41,1 & 15,4 & 8,4 \\
\hline $\begin{array}{l}\text { Participación en eventos de puertas abiertas del CSIC u } \\
\text { otros centros similares }\end{array}$ & 46,7 & 39,9 & 8,5 & 4,9 \\
\hline Participación en "Semanas de la ciencia" & 46,9 & 40 & 9,1 & 4 \\
\hline Publicación de artículos en prensa & 54,0 & 34,1 & 6,2 & 5,7 \\
\hline $\begin{array}{l}\text { Elaboración de contenidos para páginas webs de } \\
\text { carácter divulgativo }\end{array}$ & 51,7 & 38,8 & 6,2 & 3,3 \\
\hline $\begin{array}{l}\text { Cursos o conferencias en colegios de enseñanza } \\
\text { primaria o secundaria }\end{array}$ & 68,2 & 22,9 & 5,8 & 3,1 \\
\hline Participación en programas de radio o televisión & 65,7 & 26 & 6,2 & 2,1 \\
\hline Participación en documentales u otros videos científicos & 68,7 & 27,4 & 3,4 & 0,5 \\
\hline
\end{tabular}

La actividad más frecuente es la publicación de libros de texto y revistas de divulgación (en los últimos 3 años, un $77 \%$ ha participado al menos una vez, frente a un $23 \%$ que no lo ha hecho nunca). Le siguen la participación en conferencias y mesas redondas (un $65 \%$ ha participado alguna vez). A bastante distancia se encuentra la participación en las semanas de la ciencia y en otros eventos de puertas abiertas ( $53 \%$ en cada uno de ellos). Las actividades en las que más de la mitad de los investigadores encuestados no ha participado ninguna vez son la elaboración de contenidos web divulgativos $(51,7 \%)$, la publicación de artículos de prensa $(54,0 \%)$, los programas de radio o televisión $(65,7 \%)$, los documentales científicos $(68,7 \%)$ y los cursos y conferencias en colegios o centros de enseñanza $(68,2 \%)$.

Por su parte, las actividades en las cuales los investigadores han participado con más frecuencia (4 o más veces) son la publicación de textos en libros y revistas de divulgación $(28,9 \%)$, las conferencias o mesas redondas de divulgación $(23,8 \%)$ $y$, en menor medida, los cursos o conferencias en colegios de enseñanza primaria o secundaria, la participación en programas de radio o televisión y la participación en documentales u otros videos científicos (menos del $9 \%$ en todos los casos). La participación en eventos organizados por el organismo (puertas abiertas o semanas de la ciencia) se encuentran en una situación intermedia.

\subsection{Análisis factorial}

En primer lugar se ha realizado un análisis factorial con el propósito de observar pautas de asociación entre actividades, así como de reducción de datos dirigidos a construir indicadores sintéticos que sean apropiados para realizar los posteriores análisis. Para ello se ha empleado un análisis de componentes principales con una rotación Varimax (con normalización Kaiser), extrayendo factores con autovalores de 1,0 o superior (Hair y otros, 1998). Así mismo, se han codificado las respuestas sobre frecuencia de participación en las actividades de divulgación de la siguiente manera: $1=$ 'nunca'; $2=$ 'en algunas ocasiones'; 3 = 'a menudo'; y $4=$ 'muy a menudo'.

La variable referida a "elaboración de contenidos para páginas web" no se ha incluido en el análisis factorial debido a que el valor de su comunalidad era muy bajo $(0.320)^{5}$. Los resultados del análisis factorial contenidos en la tabla III permiten clasificar las ocho actividades de divulgación en dos factores que se interpretan por su coherencia con la división entre actividades que normalmente se desarrollan de manera individual, dado que los investigadores pueden llevarlas a cabo sin la intervención directa del organismo, y aquéllas realizadas normalmente con el apoyo o la colaboración de una institución, ya sea la propia institución de trabajo u otras interesadas en organizar o promocionar eventos de divulgación. Esta división tiene algunas coincidencias con estudios que clasifican las actividades de acuerdo con otros criterios. Por ejemplo, Rey-Rocha y otros (2006) tienen en cuenta los sistemas de audiencia. Distinguen entre las actividades individuales, que no requieren presencia de audiencia, de otras que requieren de un auditorio y de las de carácter institucional que requieren contacto con el público. Resulta difícil realizar una división clara entre el carácter individual, grupal o institucional de cada actividad debido a que una misma acción se puede realizar en dis- 
tintos contextos. Por ello, a partir de los estudios existentes, la estrategia de este trabajo ha sido optar por realizar un análisis factorial que ofrezca una primera pista sobre cómo se agrupan las actividades para luego comprobar su sentido. A pesar de que este procedimiento no permite detectar todas las posibles combinaciones de comportamientos, se han encontrado notables similitudes con las clasificaciones que aplican criterios lógicos.

En resumen, los dos grupos son los siguientes: (i) actividades divulgativas de carácter individual, que incluyen la publicación de textos en libros y revistas de divulgación, artículos de prensa, participación en documentales, las conferencias y mesas redondas de divulgación, y la participación en programas de radio o televisión; (ii) actividades divulgativas de carácter institucional, que incluyen la participación en cursos y conferencias en colegios, en semanas de la ciencia, y en eventos de puertas abiertas del CSIC. En el primer caso se trata de actividades que no requieren de la intervención del organismo, donde los investigadores pueden participar a título individual, mientras que en la segunda son actividades organizadas por el propio CSIC o por otras organizaciones científicas o universitarias.
Los dos factores representan un total del $57 \%$ de la varianza explicada, lo que se considera un resultado satisfactorio en los estudios de ciencias sociales (Hair y otros, 1998). Otro aspecto a considerar es la fiabilidad de los factores, es decir, el grado de consistencia entre las variables (ítems) que conforman cada factor. Una medida frecuente para verificar la fiabilidad de los factores es el alfa de Cronbach. Los dos factores (individual e institucional) tienen un alfa de Cronbach superior a 0,7 (tabla III), resultado que indica un alto nivel de fiabilidad de los factores (Field, 2009).

A partir de este resultado se han calculado dos indicadores sintéticos, correspondientes a actividades de divulgación de carácter individual e institucional. El procedimiento ha consistido en calcular el promedio de las puntuaciones ${ }^{6}$ de cada grupo de variables agrupadas en el análisis factorial, dando lugar a dos indicadores continuos (divulgación individual e institucional) cuyos descriptivos se presentan en la tabla IV. Estos dos indicadores son empleados en la siguiente sección para analizar si la participación de los investigadores en estos dos tipos de actividades de divulgación varía en función del área de conocimiento a la que pertenecen.

Tabla III. Análisis factorial. Actividades de divulgación de carácter individual e institucional

\begin{tabular}{|c|c|c|c|}
\hline \multirow{2}{*}{ Actividades de divulgación } & \multicolumn{2}{|c|}{ Factores } & \multirow{2}{*}{$\begin{array}{c}\text { a de } \\
\text { Cronbach }\end{array}$} \\
\hline & Individual & Institucional & \\
\hline Publicación de textos en libros y revistas de divulgación & 0,632 & 0,014 & \multirow{5}{*}{0,740} \\
\hline Publicación de artículos en prensa & 0,698 & 0,148 & \\
\hline Participación en documentales u otros videos científicos & 0,632 & 0,264 & \\
\hline Participación en conferencias o mesas redondas de divulgación & 0,720 & 0,191 & \\
\hline Participación en programas de radio o televisión & 0,757 & 0,166 & \\
\hline Cursos o conferencias en colegios de enseñanza primaria o secundaria & 0,381 & 0,574 & \multirow{3}{*}{0,755} \\
\hline Participación en "Semanas de la ciencia" & 0,154 & 0,880 & \\
\hline $\begin{array}{l}\text { Participación en eventos de puertas abiertas del CSIC u otros centros } \\
\text { similares }\end{array}$ & 0,066 & 0,896 & \\
\hline Varianza explicada & \multicolumn{2}{|c|}{$57,674 \%$} & \\
\hline Autovalor & 1,321 & & \\
\hline
\end{tabular}

Tabla IV. Resultados descriptivos de las actividades de divulgación de carácter individual e institucional

\begin{tabular}{lccccc}
\hline Factores & Items del indicador & Mínimo & Máximo & Media & Desviación típica \\
\hline Divulgación individual & 5 items & 1 & 4 & 1,72 & 0,56 \\
\hline Divulgación institucional & 3 items & 1 & 4 & 1,62 & 0,64 \\
\hline
\end{tabular}




\subsection{La influencia del área científica en las actividades de divulgación}

La comparación del grado de participación de los investigadores en actividades de divulgación en función del área científica se ha realizado mediante un contraste ANOVA (test de Duncan). Este análisis permite crear grupos (subconjuntos homogéneos) basados en comparación de medias. La hipótesis nula que se verifica en este test es la igualdad de medias entre diferentes áreas científicas para los indicadores de divulgación individual y divulgación institucional. A saber, si se encuentran diferencias estadísticamente significativas entre las medias correspondientes a diferentes áreas, se pueden clasificar estas áreas en diferentes subconjuntos; de lo contrario, estas áreas pertenecen al mismo subconjunto.

Los resultados del contraste ANOVA permiten identificar 3 subconjuntos homogéneos de áreas científicas que son significativamente diferentes en cuanto a la participación de los investigadores en actividades de divulgación individual (tabla V). En el caso de la participación de los investigadores en actividades de divulgación de carácter institucional, los resultados también sugieren la presencia de 3 subconjuntos homogéneos de áreas científicas que son estadísticamente diferentes (tabla VI).

Por lo que se refiere a las actividades de divulgación individual, la media de la participación de los investigadores está comprendida entre 1,98 para el área de humanidades y ciencias sociales y 1,56 para ciencia y tecnologías químicas. Humanidades y ciencias sociales es la única área incluida en el subconjunto 1 , es decir, en el subconjunto que presenta una mayor puntuación media de participación. Este resultado indica que los investigadores del área de humanidades y ciencias sociales son los que más participan, en promedio, en actividades de divulgación individual, en comparación con los investigadores de las otras siete áreas científicas. Por otra parte, no se encuentran diferencias significativas en las medias de los investigadores de las siguientes áreas: recursos naturales, ciencia y tecnología de alimentos, ciencias agrarias, y ciencia y tecnologías físicas. Estas cuatro áreas científicas pertenecen al subconjunto 2 cuyas medias están comprendidas entre 1,83 y 1,75. Por último, el subconjunto 3, del que forman parte los investigadores de las áreas de ciencia y tecnología de materiales, biología y biomedicina, y ciencia y tecnologías químicas, agrupa a los investigadores que menos participan en este tipo de actividades, no habiendo diferencias de medias estadísticamente significativas entre ellas.

Cuando el ejercicio se realiza para las actividades de divulgación institucional, la media de la participación de los investigadores está comprendida entre 1,76 para el área de ciencia y tecnologías químicas y 1,42 para el área de humanidades y ciencias sociales. Los resultados sugieren un primer subconjunto homogéneo de áreas científicas (ciencia y tecnologías químicas, ciencias agrarias, ciencia y tecnología de alimentos, y ciencia y tec-

Tabla V. Comparación de medias de la participación de los investigadores en divulgación de carácter individual (post hoc-Duncan test) $\dagger$

\begin{tabular}{|c|c|c|c|c|c|}
\hline \multirow{2}{*}{\multicolumn{2}{|c|}{$\begin{array}{l}\text { Indicador de participación en actividades de divulgación de carácter } \\
\text { individual } \\
\text { Áreas científicas }\end{array}$}} & \multicolumn{4}{|c|}{ Subconjunto para $a=0,05$} \\
\hline & & \multirow{2}{*}{ N } & \multirow{2}{*}{1,98} & \multirow[t]{2}{*}{2} & \multirow[t]{2}{*}{3} \\
\hline • & Humanidades y ciencias sociales & & & & \\
\hline • & Recursos naturales & 277 & & 1,83 & \\
\hline • & Ciencia y tecnología de alimentos & 128 & & 1,82 & \\
\hline • & Ciencias agrarias & 203 & & 1,77 & \\
\hline • & Ciencia y tecnologías físicas & 204 & & 1,75 & \\
\hline • & Ciencia y tecnología de materiales & 199 & & & 1,63 \\
\hline • & Biología y biomedicina & 244 & & & 1,57 \\
\hline • & Ciencia y tecnologías químicas & 208 & & & 1,56 \\
\hline \multicolumn{2}{|c|}{ Significatividad ${ }^{++}$} & & 1,000 & 0,216 & 0,250 \\
\hline
\end{tabular}

† El test de Duncan compara medias de grupos en subconjuntos homogéneos cuando se asumen varianzas iguales.

†† Cuando la prueba de significación está por encima del umbral =0,05, la hipótesis nula (no diferencias de medias) no puede ser rechazada. 
nologías físicas) entre cuyas medias no hay diferencias significativas y que son las que presentan mayores frecuencias medias de participación de los investigadores en divulgación institucional. El subconjunto 2 (que comprende valores medios entre 1,67 y 1,53 ) contiene un grupo de seis áreas científicas cuyas medias no difieren estadísticamente entre ellas: ciencias agrarias, ciencia y tecnología de alimentos, ciencia y tecnologías físicas, ciencia y tecnología de materiales, recursos naturales, y biología y biomedicina. Como puede observarse, las áreas de ciencias agrarias, ciencia y tecnología de los alimentos y ciencia y tecnologías físicas pertenecen tanto al primer subconjunto como al segundo. Este resultado sugiere que las medias de estas áreas no difieren de las medias de la de ciencia y tecnologías químicas, ni de las tres áreas situadas inmediatamente a continuación. Finalmente, se puede distinguir un tercer subconjunto homogéneo formado por las áreas de biología y biomedicina y humanidades y ciencias sociales, con valores medios entre 1,53 y 1,42. Nuevamente, el área de biología y biomedicina pertenece al segundo y tercer subconjunto, lo cual se puede interpretar como en el caso anterior. En este sentido, la media de participación de los investigadores del área de biología y biomedicina es inferior a la de las áreas del subconjunto 1 . Sin embargo, no difiere de las medias de los investigadores de las áreas incluidas en los subconjuntos 2 y 3 . Por otro lado, los investigadores del área de humanidades y ciencias sociales participan menos (en promedio) que los investigadores del resto de áreas científicas (con la excepción del área de biología y biomedicina, con la que no se encuentran diferencias significativas).

El contraste ANOVA ha permitido identificar la existencia de diferentes prácticas de participación en los distintos tipos de actividades de divulgación analizados: individual e institucional en función del área científica. En este resultado conviene destacar dos hechos relevantes. Por un lado, los investigadores del área de humanidades y ciencias sociales muestran una mayor propensión a realizar actividades basadas en sus capacidades e iniciativas personales, pues son los que tienen una mayor participación (en promedio) en actividades de divulgación individual y, a la vez, son los que presentan un promedio menor de participación en actividades de divulgación institucional. En la otra cara de la moneda se encuentran los investigadores del área de ciencia y tecnologías químicas, que lideran la participación en actividades de divulgación institucional pero apenas participan en actividades individuales.

En el conjunto de las áreas sorprende que el área de recursos naturales, en la que se encuentran centros como el Museo de Ciencias Naturales o el Real Jardín Botánico, que tienen unidades de divulgación propias y desarrollan múltiples actividades, no aparezca en posiciones más destacadas en las actividades institucionales. También es digno de mención que el área de biología y biomedicina se encuentra en ambos casos en la zona de valores más bajos. Este caso plantea retos para los responsables de estas actividades, pues es un ámbito

Tabla VI. Comparación de medias de la participación de los investigadores en divulgación de carácter institucional (post hoc-Duncan test) $\dagger$

\begin{tabular}{|c|c|c|c|c|}
\hline \multirow{2}{*}{$\begin{array}{l}\text { Indicador de participación en actividades de divulgación de carácter } \\
\text { institucional } \\
\text { Áreas científicas }\end{array}$} & \multicolumn{4}{|c|}{ Subconjunto para $a=0,05$} \\
\hline & $\mathrm{N}$ & 1 & 2 & 3 \\
\hline - Ciencia y tecnologías químicas & 207 & 1,76 & & \\
\hline - Ciencias agrarias & 203 & 1,67 & 1,67 & \\
\hline - Ciencia y tecnología de alimentos & 127 & 1,67 & 1,67 & \\
\hline - $\quad$ Ciencia y tecnologías físicas & 203 & 1,64 & 1,64 & \\
\hline - $\quad$ Ciencia y tecnología de materiales & 200 & & 1,62 & \\
\hline - $\quad$ Recursos naturales & 277 & & 1,60 & \\
\hline - Biología y biomedicina & 244 & & 1,53 & 1,53 \\
\hline - Humanidades y ciencias sociales & 115 & & & 1,42 \\
\hline Significatividad ${ }^{++}$ & & 0,085 & 0,054 & 0,096 \\
\hline
\end{tabular}

† El test de Duncan compara las medias de grupos en subconjuntos homogéneos cuando se asumen varianzas iguales.

†† Cuando la prueba de significación está por encima del umbral $=0,05$, la hipótesis nula (no diferencias de medias) no puede ser rechazada. 
en el que los avances científicos son notables y a la vez de alto interés para los ciudadanos, donde en ocasiones se plantean conflictos para los que los ciudadanos necesitan información a la hora de conformar criterios de valoración. Es, sin duda, un área que requeriría un análisis profundo y el diseño de estrategias específicas.

\section{CONCLUSIONES}

Los resultados muestran que, en términos generales, la tasa de inactividad de los científicos del CSIC en actividades de divulgación es muy baja (menos del 6\%), y el nivel de actividad general es alto: más de un $75 \%$ ha participado en 3 o más actividades diferentes. Estos resultados son similares a otras investigaciones realizadas en el contexto español. Por otra parte, estudios realizados en otro centro similar -el CNRS francés- (Jensen, 2011), aunque no sean estrictamente comparables, pues no se han llevado a cabo con los mismos criterios, muestran resultados diversos, con niveles de inactividad superiores al $40 \%$ y siempre menos de una acción por año.

El análisis factorial de los diversos tipos de actividades ha permitido dividir las actividades de divulgación en dos grandes grupos -actividades individuales e institucionales-, habiéndose podido observar diferencias entre áreas del conocimiento. En general, los investigadores de ciencias humanas y sociales tienen mayor propensión hacia las actividades de carácter individual, mientras que los investigadores de las disciplinas experimentales participan más en actividades promovidas o realizadas por las instituciones. No obstante, las diferencias en este último grupo no son tan acusadas, pues hay un área, la de biología y biomedicina, que presenta valores medios bajos en ambas categorías. Además, algunas de las acciones de divulgación de carácter institucional no se dirigen exclusivamente a divulgar resultados sino también a comunicar al público los procesos de investigación, a mostrar las instalaciones en las que se llevan a cabo, o a explicar fenómenos o conceptos científicos de actualidad, lo cual puede explicar en parte las diferencias observadas en las áreas experimentales (por ejemplo, el año 2011 fue el Año de la Química, acontecimiento que puede haber influido en la gran participación del colectivo de químicos en actividades divulgativas de carácter institucional). Por consiguiente, no cabe delimitar nítidamente dos culturas, la humanística y la de las ciencias experimentales, porque sólo en el caso de las actividades de divulgación individuales los investigadores de humanidades y ciencias sociales se encuentran en un grupo aislado, pero los investigadores de las áreas experimentales se dividen en dos grupos, por lo que su comportamiento no es monolítico.

Los resultados sugieren, por una parte, que los investigadores de ciencias humanas y ciencias sociales tienen más interiorizada su aportación al desarrollo de la cultura de los ciudadanos, pues desarrollan más en solitario sus actividades de divulgación, mayoritariamente por los cauces que han utilizado tradicionalmente para diseminar socialmente sus conocimientos (publicación de libros, artículos en prensa, participación en documentales, conferencias,...). Por otra parte, los investigadores de ciencias experimentales han asumido este rol con posterioridad y en gran medida gracias a los esfuerzos realizados desde las instancias políticas e institucionales, pues destaca su participación en las actividades organizadas por alguna institución (semanas de la ciencia, jornadas de puertas abiertas). A este respecto, los resultados permiten concluir que las políticas de promoción de la cultura científica de tipo institucional desarrolladas en el último decenio han tenido cierto éxito dinamizando al colectivo de los científicos experimentales y no tanto en el caso de las ciencias humanas y sociales, que desarrollan sus propias dinámicas de divulgación al margen de las instituciones. Asimismo, los resultados del trabajo pueden ayudar a diseñar acciones específicas para las diversas áreas, a fin de movilizar a los colectivos que actualmente están menos implicados hacia aquellas actividades que se consideren de mayor impacto o interés social.

Estos resultados aconsejan realizar estudios más detallados en esta línea de investigación. En este sentido, sería relevante explorar qué otros factores (e.g. características socio-demográficas, situación profesional y carrera profesional del investigador, etc.) determinan la participación de los investigadores en distintos tipos de actividades de divulgación y determinar si estos factores difieren para cada tipo de actividad.

\section{AGRADECIMIENTOS}

Este artículo utiliza los datos producidos en el proyecto "Una aproximación al impacto socioeconómico de las actividades del CSIC", realizado a instancias de la Presidencia del CSIC a través de su Vicepresidencia Adjunta de Transferencia de Conocimiento (VATC). Lo han Ilevado a cabo conjuntamente el Instituto de Estudios Sociales AvanzadosIESA e INGENIO (CSIC-UPV). Los autores reconocen el trabajo colectivo de los demás miembros del equipo que han intervenido en la elaboración de la encuesta a investigadores, especialmente de Ignacio Fernández de Lucio, Leticia Rodríguez Brey, Carmen Merchán Hernández, Oihana Valmaseda Andía, Elena Espinosa de los Monteros, Pablo D’Este, Jaider Vega, Liney Majarrés, Antonio Gutiérrez y Manuel Pérez Yruela. La responsabilidad en la interpretación recae únicamente en los autores. Así mismo, se agradece a todos los investigadores que han respondido a la encuesta por su contribución a la realización de este proyecto. Se agradecen también las aportaciones de Carmen Guerrero Martínez, Laura Ferrando (Vicepresidencia Adjunta de Cultura Científica) e Isabel Bernal (responsable de ConCiencia) del CSIC, que con sus valiosos comentarios y sugerencias han colaborado en la me- 
jora de la versión revisada del artículo. Por último, agradecer a los dos revisores anónimos sus valiosos comentarios que han resultado de gran ayuda para la mejora del artículo.

\section{NOTAS}

[1] http://www.unesco.org/science/wcs/eng/declaration_e.htm

[2] REAL DECRETO 1730/2007, de 21 de diciembre, por el que se crea la Agencia Estatal Consejo Superior de Investigaciones Científicas y se aprueba su Estatuto (BOE del 14 de enero de 2008). Artículo 5 -funciones-, apartado h: Fomentar la cultura científica en la sociedad; apartado p: Colaborar en la actualización de conocimientos en ciencia y tecnología del profesorado de enseñanzas no universitarias).

[3] Los Quinquenios, o Méritos Investigadores del Complemento Específico, son un complemento salarial que pueden solicitar los investigadores funcionarios voluntariamente por cada cinco años de trabajo. Si las solicitudes son evaluadas favorablemente, el investigador obtiene un pequeño complemento salarial que se incorpora a la nómina de manera estable.

[4] Inicialmente se disponía de 4.275, pero se han eliminado los que corresponden a personas que trabajan en la organización central del CSIC desempeñando tareas de planificación y gestión de la investigación.

[5] La varianza explicada del análisis factorial mejora del $54 \%$ al $57,6 \%$ al no incluir la variable "elaboración de contenidos para páginas web".

[6] Las puntuaciones de las variables corresponden a los valores nuevos que se han asignado a cada una de las respuestas proporcionadas por los investigadores (1= 'nunca'; $2=$ 'en algunas ocasiones'; 3 = 'a menudo'; y 4 = 'muy a menudo'). Por lo tanto al calcular el promedio, se obtienen valores comprendidos entre 1 y 4 , tal y como se detalla en la tabla IV.

\section{BIBLIOGRAFÍA}

Abbott, A. (2001). Chaos of disciplines. Chicago; University Press.

Amara, N. ; Ouimet, M. ; Landry, R. (2004). New evidence on instrumental, conceptual, and symbolic utilization of university research in government agencies. Science Communication, vol. 26 (1), 75-106. http://dx.doi.org/10.1177/1075547004267491

Bauer, M. W.; Jensen, P. (2011). The mobilization of scientists for public engagement. Public Understanding of Science, vol. 20 (1), 3-11. http://dx.doi. org/10.1177/0963662510394457

Bernal, I.; Ponsati Obiols, A. (2011). Placing Digital. CSIC as a key element within CSIC's Scientific Information Management System. e-colabora: Revista de ciencia, educación, innovación y cultura apoyadas por redes de tecnología avanzada, vol. 1 (2), 34-49.

Besley, J. C.;Hwa Oh, S.; Nisbet, M. (2012). Predicting scientists' participation in public life. Public Understanding of Science, (publicado en "Online First", 17 de octubre 2012 doi: 10.1177/0963662512459315).

Beyer, J. M., y Trice, H. M. (1982). The utilization process: A conceptual framework and synthesis of empirical findings. Administrative Science Quarterly, 27(4), 591-622. http://dx.doi. org/10.2307/2392533
Burns, T. W.;O'Connor, D. J.; Stocklmayer, S. M. (2003). Science Communication: A Contemporary Definition. Public Understanding of Science, vol. 12 (2), 183-202. http://dx.doi. org/10.1177/09636625030122004

CICYT (2008). Plan Nacional de Investigación Científica, Desarrollo e Innovación Tecnológica 20082011. FECYT. http://www.idi.mineco.gob.es/stfls/ MICINN/Investigacion/FICHEROS/PLAN_NACIONAL_CONSEJO_DE_MINISTROS.pdf [acceso el 4 de mayo de 2013].

Comisión Europea (2006). Science in society portal. http://ec. europa.eu/research/science-society/index.cfm?fuseaction $=$ public.topic\&id $=1221$ [acceso el 4 de mayo de 2013].

CSIC (2012). Memoria anual 2011. Madrid: CSIC.

Delanty, G. (2001). Challenging knowledge: The university in the knowledge society. Buckingham, UK; Open University Press.

FECYT (2003). Memoria de actividades 2002: Madrid. http://www.fecyt.es/fecyt/docs/tmp/1674903526. pdf [acceso el 4 de mayo de 2013].

FECYT (2011). Diez años de divulgación científica en España. Fundación Española para la Ciencia y la Tecnología: Madrid. http://www.fecyt.es/fecyt/ docs/tmp/1081372493.pdf [acceso el 3 de noviembre de 2013].

FECYT (varios años). Estrategia Nacional de Ciencia y Tecnología. Comisión Interministerial de Ciencia y Tecnología. http://www.idi.mineco.gob.es/stfls/MICINN/investigacion/FICHEROS/Encyt.pdf

Field, A. P. (2009). Discovering statistics using SPSS. Thousand Oaks, CA; Sage publications.

Garland, R. (2012). The humanities: plain and simple. Arts and Humanities in Higher Education, 11(3), 300-312. http://dx.doi. org/10.1177/1474022212438754

Guston, D. H.; Keniston, K. (editores.) (1994) The fragile contract: university science and the Federal Government, Cambridge, MIT Press.

Hair, J. F.; Anderson, R. E.; Tatham, R. L. ; Black, W. C. (1998). Multivariate data analysis (5a Edición) New Jersey; Prentice Hall.

Jacobson, N. ; Butterill, D. ; Goering, P. (2004). Organizational factors that influence University-Based Researchers' Engagement in Knowledge Transfer activities. Science Communication, vol. 25 (3), 246-259. http://dx.doi.org/10.1177/1075547003262038

Jensen, P. (2011). A statistical picture of popularization activities and their evolutions in France. $\mathrm{Pu}-$ blic Understanding of Science, vol. 20 (1), 26-36. http://dx.doi.org/10.1177/0963662510383632

Jensen, P.; Rouquier, J. B.; Kreimer, P.; Croissant, Y. (2008). Scientists who engage with society perform better academically. Science and Public Policy, vol. 35 (7), 527-541.

Kalleberg, R. (2000). Universities: Complex bundle institutions and the projects of enlightenment. Comparative Social Research, vol. 19, 219-255. http:// dx.doi.org/10.1016/S0195-6310(00)80026-5

Kingston, J. (2012). Choosing a Knowledge Dissemination Approach. Knowledge and Process Management, vol. 19 (3), 160-170. http://dx.doi. org/10.1002/kpm.1391 
Kunth, D. (1992). La place du chercheur dans la vulgarisation scientifique. Lyon: Départment culture scientifique et technique. http://sciences-medias. ens-Iyon.fr/scs/IMG/pdf/rapport_Kunth-3.pdf [acceso el 7 de octubre de 2013].

Kyvik, S. (2005). Popular science publishing and contributions to public discourse among university faculty. Science communication, vol. 26 (3), 288-311. http://dx.doi.org/10.1177/1075547004273022

León, S. (2011). La polémica Snow-Leavis. En Historia Siglo XXI, 20-jul-2011. http://suite101.net/article/ la-polemica-snow-leavis-a60974 [acceso el 28 de Abril de 2013].

López Cerezo, J.; Verdadero, C. (2003). Introduction: science, technology and society studies-from the European and American north to the Latin American south. Technology in Society, vol. 25 (2), 153-170. http://dx.doi.org/10.1016/S0160791X(03)00027-7

Martín-Sempere, M. J.;Rey-Rocha, J.;Garzón-García, B.;Tigeras-Sánchez, P.; Pérez-del-Val, J. (2004). Los científicos del CSIC ante el público: experiencia en la Feria Madrid por la Ciencia. Madrid: CSIC.

Martín-Sempere, M. J.; Garzón-García, B.; Rey-Rocha, J. (2008). Scientists' motivation to communicate science and technology to the public: surveying participants at the Madrid Science Fair. Public Understanding of Science, vol. 17 (3), 349-367. http://dx.doi.org/10.1177/0963662506067660

OCDE. (1996). The Knowledge-based Economy. París: OCDE.

Olmos-Peñuela, J., Benneworth, P., y Castro-Martínez, E. (2013). Are 'STEM from Mars and SSH from Venus'?: Challenging disciplinary stereotypes of research's social value. Science and Public Policy, (publicado en "Online First", 3 de octubre 2013 doi: 10.1093/scipol/sct1071).
Pavitt, K. (1984). Sectoral patterns of technical change: towards a taxonomy and a theory. Research Policy, vol. 13 (6), 343-373. http://dx.doi. org/10.1016/0048-7333(84)90018-0

Poliakoff, E.; Webb, T. L. (2007). What factors predict scientists' intentions to participate in public engagement of science activities? Science Communication, vol. 29 (2), 242-263. http://dx.doi. org/10.1177/1075547007308009

Polt, W. ; Rammer, C. ; Gassler, H.; Schibany, A.; Schartinger, D. (2001). Benchmarking industry-science relations: the role of framework conditions. Science and Public Policy, vol. 28 (4), 247-258.

Rey-Rocha, J.;Martín-Sempere, M. J.;Garzón-García, B.; de Bustamante Gutiérrez, I. (2006). Los científicos de la Universidad ante el público: experiencia en la Feria Madrid por la Ciencia. Madrid: CSIC.

Snow, C. P. (1959). The Two Cultures and the Scientific Revolution. London; Cambridge University Press.

Snow, C. P. (1963). The Two Cultures and a Second Look. London; Cambridge University Press.

Torres-Albero, C.; Fernández-Esquinas, M.; Rey-Rocha, J.; Martín-Sempere, M. J. (2011). Dissemination practices in the Spanish research system: scientists trapped in a golden cage. Public Understanding of Science, vol. 20 (1), 12-25. http:// dx.doi.org/10.1177/0963662510382361

Weigold, M. F. (2001). Communicating Science: A Review of the Literature. Science Communication, 23(2), 164-193. http://dx.doi. org/10.1177/1075547001023002005

Ziman, J. (1995): Of one mind: The collectivization of Science, Woodbury: AIP Press. 